\title{
Author's Reply: Indications and Outcomes for Non-Trauma Emergency Laparotomy: A Comparison of Rwanda, South Africa, and the USA
}

\author{
Jennifer Rickard ${ }^{1,2} \cdot$ Kathryn $\mathrm{Chu}^{3,4,5}$
}

Accepted: 5 January 2021/Published online: 27 January 2021

(C) Société Internationale de Chirurgie 2021

We thank Prof. Atamanalp for his interest in our article [1] and appreciate his interesting "Letter to the Editor". His impressive database on sigmoid volvulus and ileosigmoid knotting $[2,3]$ significantly contributes to the literature on this topic because it highlights a disease relatively common in sub-Saharan Africa, Latin America, and Eastern Europe but less so in North America and Western Europe [4].

Thus far, the data support high fibre diet as a risk factor for sigmoid volvulus [4]. Other factors that have been implicated in sigmoid volvulus include anatomic predispositions [4]. However, questions remain. How much do environmental factors (such as high fibre diet) contribute to risk compared with genetic or anatomic factors?

Epidemiologic studies contribute to our understanding of disease processes and their inherent risk factors. They can have important implications for prevention, management and outcomes. To date, there is a great disparity in access and availability of data. Most published data come from high resource settings. Even in high resource settings, there is disparate access to information on minority

Jennifer Rickard

gehr0059@umn.edu

1 Department of Surgery, University Teaching Hospital of Kigali, Kigali, Rwanda

2 Department of Surgery, University of Minnesota, 420 Delaware St SE, MMC 195, Minneapolis, MN 55455, USA

3 Department of Surgery, University of Cape Town, Cape Town, South Africa

4 Department of Surgery, New Somerset Hospital, Cape Town, South Africa

5 Centre for Global Surgery, Department of Global Health, Stellenbosch University, Cape Town, South Africa populations. These disparities in data contribute to inequalities in cares and outcomes.

The COVID-19 pandemic has demonstrated that diseases no longer stay endemic to resource-rich or resourcepoor settings. We live in an increasingly globalized world where it is important to understand disease processes on a global scale, not just in our local population. There are growing rates of travel, immigration, and emigration. Even in our local environment, we are likely to encounter individuals from different communities, cultures, and environments. We need to be prepared to respond appropriately. For example, Minnesota is home to a large Somali community [5]. While some populations assimilate to new environments, many retain their cultural heritage where possible. Data from differing regions, as well as immigrant and refugee populations, can help us better understand the relative contributions of environmental and genetic contributions to diseases.

In summary, we must commit more equitable resources towards understanding all diseases, especially ones which are more common in lower resourced settings and amongst vulnerable populations. This would include comprehensive data collection to compare conditions across different populations with differing risk factors. Through such analyses, we can gather more information on relative risk of specific factors. Depending on the identified risk factors, interventions can be introduced to then reduce excess risk of morbidity and mortality.

Acknowledgement This manuscript has not been published elsewhere and is not under consideration for publication elsewhere.

Funding No funding was received for this letter. 


\section{Compliance with ethical standards}

Conflict of interest The authors have no disclosures or conflicts of interest to report.

\section{References}

1. Rickard J, Pohl L, Abahuje E, et al. (2020) Indications and outcomes for non-trauma emergency laparotomy: a comparison of Rwanda, South Africa, and the USA. World J Surg. https://doi.org/ 10.1007/s00268-020-05862-y
2. Atamanalp SS (2019) Sigmoid volvulus: the first one thousandcase single center series in the world. Eur J Trauma Emerg Surg 45:175-176

3. Atamanalp SS (2018) Ileosigmoid knotting: one of the largest single-center series. Pak J Med Sci 34:671-675

4. Raveenthiran V, Madiba TE, Atamanalp SS et al (2010) Volvulus of the sigmoid colon. Colorectal Dis 12:e1-17

5. Somali Refugees: Centers for Disease Control and Prevention (2018) [27 December 2021]. https://www.cdc.gov/immigrantrefu geehealth/profiles/somali/populationMovements.html.

Publisher's Note Springer Nature remains neutral with regard to jurisdictional claims in published maps and institutional affiliations. 